\title{
Immunolocalization of decorin, a small leucin-rich proteoglycan, in the normal and injured horse tendon
}

\section{O.J. Ali $\mathbb{D}$}

Department of Surgery and Theriogenology, College of Veterinary Medicine, University of Sulaimani, Sulaymaniyah, Iraq

\begin{tabular}{l} 
Article information \\
\hline Article history: \\
Received May 02, 2020 \\
Accepted July 14, 2020 \\
Available online June 23, 2021 \\
\hline Keywords: \\
SDFT \\
Proteoglycan \\
Decorin \\
Tendinopathy \\
\\
\hline Correspondence: \\
O.J. Ali \\
othman.ali@ univsul.edu.iq
\end{tabular}

\begin{abstract}
The aim of this study was to evaluate the intensity of decorin in normal and the injured equine cadaver limbs. The study was carried out using superficial digital flexor tendon (SDFT) samples harvested from equine cadaver limbs. Randomly assigned left or right equine distal forelimbs were collected in normal ( $7-14$ years old $\pm 2.86 \mathrm{SD})$ and injured (6 - 16 years old $\pm 3.64 \mathrm{SD}$ ) SDFT. Small pieces of approximately $5 \times 5 \times 3 \mathrm{~mm}$ were collected from normal and the injured areas at the mid-most metacarpal regions and fixed in $4 \%$ paraformaldehyde at room temperature. They were routinely prepared for $\mathrm{H} \& \mathrm{E}$ staining in order to confirm whether they were normal or injured. Then they were prepared for immunological assay, the primary and the secondary antibodies were prepared in a standard ratio. The results showed that the distribution of decorin was varied between fascicles and the interfascicular matrix (IFM), and the latter showed greater intensity than the fascicles. The injured tendons showed up-regulation of decorin, it was intensively expressed in all the cases of injured tendon, particularly fascicles showed significant up-regulation when compared to the normal tendon. It was concluded that decorin was varied regionally, and the injured tendons presented a significant up-regulation.
\end{abstract}

DOI: 10.33899/ijvs.2020.127017.1436, (C2021, College of Veterinary Medicine, University of Mosul.

This is an open access article under the CC BY 4.0 license (http://creativecommons.org/licenses/by/4.0/).

\section{Introduction}

Tendon is made up of a complex hierarchicaly arranged different sized fascicles, they are functioning to transfer forces from muscles to the bones, which subsequently aids various patterns of movement within the skeletal system (1$3)$. These fascicles of the equine superficial digital flexor tendon (SDFT) are outlined by the interfascicular matrix (IFM) and they are considered as the energy storing units, which are functionally equivalent to the human Achilles tendon (4). In addition to the hierarchical arrangement, different sized proteoglycans (PG) are distributed between the collagen fibers of the fascicles and the IFM, and they serve different functions during normal development and tendinopathy (5). These PGs are classified into two main groups according to their sizes; the first group is called small (40-60 kDa) leucine-rich PGs (SLRP), including decorin (most abundant), keratocan, biglycan, lumican and fibromodulin. The SLRP have a Leucine-rich small core protein, consist of a sequence of about 20-30 amino-acids that containing conserved traces of leucine, which bind to the collagen fibrils and play a role in the process of fibrillogenesis. Although, it has been recorded that the most prominent SLRP is the decorin and constitute $80 \%$ of the total PG content particularly in the tensional region of tendon (6-8). The second group is called modular PGs or Lecticans including aggrecan and versican, which are larger than SLRP, they are rich in chondroitin sulphate and dermatan sulphate (6). In this group, PGs interact with the collagen fibers and enable them to resist high compressive and tensional forces $(9,10)$.

Decorin is the most abundant SLRP and accounting for approximately $80 \%$ of the total PGs content (11). It binds to almost all the types of collagen fibrils either through its core protein or the glycosaminoglycans (GAG) chains such as chondroitin sulphate or dermatan sulphate, and produced by 
different cells such as fibroblasts, chondrocytes, endothelial cells and smooth muscle cells, thus it plays important role in different tissue rather than the tendon. It serves to regulate fibrillogenesis, act as a bridge between the collagen fibrils, preserves their alignment and associated with the collagen turnover. Moreover, it enhances cell proliferation, modulate growth factor activities and stimulate immune responses (1214). In the injured tendon, tenocytes and the collagen fibers undergo variable degenerative change, such as presence of pyknotic nuclei, necrosis, haemorrhage and collagen fibrils disintegration. In addition to the inflammatory reaction, there is also a quantitative up-regulation of aggrecan, decorin, collagen-II, GAG, tenocytes and matrix metalloproteinase activity $(15,16)$. From these micromolecular alterations, decorin is the most common types that involved during SDFT injury but they are not regionally specified (11). However, in the human the Achilles tendon, decorin up regulation in tendinopathic patient showed no significant differences when compared to the normal tendon (17-19). For this reason, the current study aimed to show how decorin is distributed within the intact SDFT and how it altered during injury.

\section{Material and methods}

\section{Samples collections}

The study was carried out using SDFT samples harvested from equine cadaver limbs. Samples from the forelimb were collected because of the fact that most of the injuries were affected SDFT of fore limbs as a result of a heavy loads $(65 \%$ of body weight) on the forelimbs during racing. The samples were randomly assigned from the right and left equine distal forelimbs, from normal and injured SDFT tendons. Collection included limbs of different aged from four injured tendon (6 -16 years old $\pm 3.64 \mathrm{SD})$ and four intact tendons (7-14 years old $\pm 2.86 \mathrm{SD}$ ) (Table 1). The samples were dissected free from the surrounding soft tissues and a pieces of approximately $5 \times 5 \times 3 \mathrm{~mm}$ were collected from the core of normal and the injured tendons of the midmost metacarpal region and they were fixed in paraformaldehyde (4\%) at room temperature.

\section{Haematoxylin and Eosin staining $(\mathrm{H} \& \mathrm{E})$}

The samples were routinely prepared for H\&E staining, the protocol of $H \& E$ staining was performed according to the procedure described in the literatures (20).

\section{Immunohistochemistry (IHC)}

For this study a $5 \mu \mathrm{m}$ thin sections were cut and collected on the polylysine slides. The tissue sections were deparaffinised (dewaxed) in two xylenes (10 minutes for each) and rehydrated in a series of descending concentrated ethanol (Two 100\%, Two 95\%, Two 85\%, 70\%) then to distilled water for 3 minutes. In order to inhibit endogenous peroxidase reaction of the tissue, the sections were pre- treated with $3 \% \mathrm{H}_{2} \mathrm{O}_{2}$ for 10 minutes and then two times washed in distilled water, 10 minutes per each time. Then the SDFT sections were treated with chondroitinase ABC order to remove chondroitin and dermatan sulphates side chains from the core PGs in a concentration of $0.5 \mu 1$ chondroitinase $\mathrm{ABC}$ in $1 \mathrm{ml}$ of Tris-Hcl for 30 minutes at a $\mathrm{pH}$ of 7.2-7.4, which then washed in Tris buffer saline-Tween (TBST) $(0.05 \%)$ for 5 minutes. The SDFT sections then treated with goat serum (20\% in TBST) for 1 hours at room temperature $\left(25^{\circ} \mathrm{C}\right)$, then the goat serum was discarded and the primary antibody (mouse monoclonal IgG, Abcam 5E8E7) were applied at the concentration of 1:50 were diluted in 5\% goat serum with TBST. Sections were incubated with primary antibody overnight at $4^{\circ} \mathrm{C}$ in a refrigerator. A day after, the sections were washed with TBST for 5 minutes and then the secondary peroxidase-conjugated antibody (Goat polyclonal anti-mouse IgG, Sigma-Aldrich Ltd A4416) were applied at concentration of 1:50 in $20 \%$ diluted goat serum in TBST and kept in a hybridizer for 1 hour at room temperature $\left(25^{\circ} \mathrm{C}\right)$. The sections were removed from the hybridizer, washed in TBST for 5 minutes. The peroxidase-conjugated antibody on the SDFT sections were stained with 3,3diaminobenzidine (Sigma Aldrich) for 1 minute and then washed with distilled water for 5 minutes three times. The cellular nuclei of sections were counterstained for only few seconds with Mayer's haemalum, which then gently washed under running tap water and dehydrated by passing through ascending concentrated ethanol (70\%, Two $85 \%$, Two $95 \%$ Two 100\%) 5 minutes for each. Finally, the sections were cleaned in two xylene (10 minutes for each), then mounted with Di-n-butyl phthalate in xylene (DPX) and cover slipped $(8,21)$.

Table 1: Shows ages and limbs and the numbers of equine SDFT samples were taken from intact and the injured limbs

\begin{tabular}{|c|c|c|c|c|}
\hline & No & Age/ Years & No. of SDFT & Sides \\
\hline \multirow{4}{*}{ 莺富 } & 1 & 6 & 1 & Right \\
\hline & 2 & 9 & 1 & Left \\
\hline & 3 & 11 & 1 & Left \\
\hline & 4 & 16 & 1 & Left \\
\hline \multirow{4}{*}{ 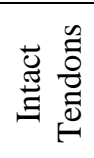 } & 1 & 7 & 1 & Right \\
\hline & 2 & 7 & 1 & Left \\
\hline & 3 & 9 & 1 & Right \\
\hline & 4 & 14 & 1 & Right \\
\hline
\end{tabular}

\section{Assessment of decorin immunostaining intensity}

For assessment of the intensity of the decorin immunostaining reaction within the normal and the injured tendon, three independents separately observers were blindly estimated the images. The intensity of immunostaining was graded from 0 to 5 according to the degree of intensity, were $0,1,2,3,4$ and 5 representing no, sight, mild, moderate, complete and very intensive staining respectively (8). 


\section{Statistical analysis}

All results were presented as mean \pm standard deviation (SD) and GraphPad prism (version 5) software were used for statistical analysis, un-paired $\mathrm{T}$ test were used with a $(\mathrm{P}<0.05)$ was considered statistically significant.

\section{Results}

The histological section of the normal tendon is composed of dense collagen fibers termed fascicles, which were outlined by the IFMs. There is a small number of tenocytes that are regularly distributed between the collagen fibers along their longitudinal axis. Although, the IFM contained a larger number of dis-organised cells (Figure 1 A). During tendinopathy the collagen fibers of both fascicles and the IFM become disrupted and disorganised with their cellular alteration. The cells are altered their orientation and shapes when compared to the intact tendon (Figure $1 \mathrm{~B}$ ). However, the ground substances are altered in the injured tendon but it could not be feasible to be outlined by using $\mathrm{H} \& \mathrm{E}$. The H\&E staining were used as guide for identifying the intact and the injured tendon before starting to immunolocalize decorin within the intact and the injured tendon. With this staining it was confirmed that the selected tendons were definitely normal in the first group and injured in the second group in spite of their clinical history.

In the immunolocalization study of the decorin using mouse monoclonal $\operatorname{IgG}$ which is specific and accurate to react to the core proteins of decorin. All the SDFT samples were shown active positive reaction for decorin immunostaining, but its distribution was varied regionally within the normal tendon and the injured tendons (Figure 2). All the normal SDFTs were showed apparent distribution of decorin within the entire tendon tissue including fascicles and the IFM, but the intensity reaction of decorin was varied between fascicles and the IFMs. The IFMs showed a greater intensity of immunostaining reaction in contrast to the fascicles. The IFMs were outlining the fascicles and it composed of irregular fibrous connective tissue, were contained blood and a large number of irregularly arranged cells. Where, the decorin intensity immunostaining appeared around the cellular elements, collagen fibrils and the walls of the blood vessels. Thus, the IFM of the normal SDFTs were showed a significant scoring intensity than the fascicles. The average intensity immunostaining of decorin in the IFMs and fascicles with their standard deviation (SD) were $3.16( \pm 0.31$ $\mathrm{SD})$ and $1.6( \pm 0.37 \mathrm{SD})$ respectively. Statistically, it was found a significant difference between the IFM and the fascicles, using unpaired $\mathrm{T}$ test with a $\mathrm{P}$ value $<0.5$ (Figure $3)$.

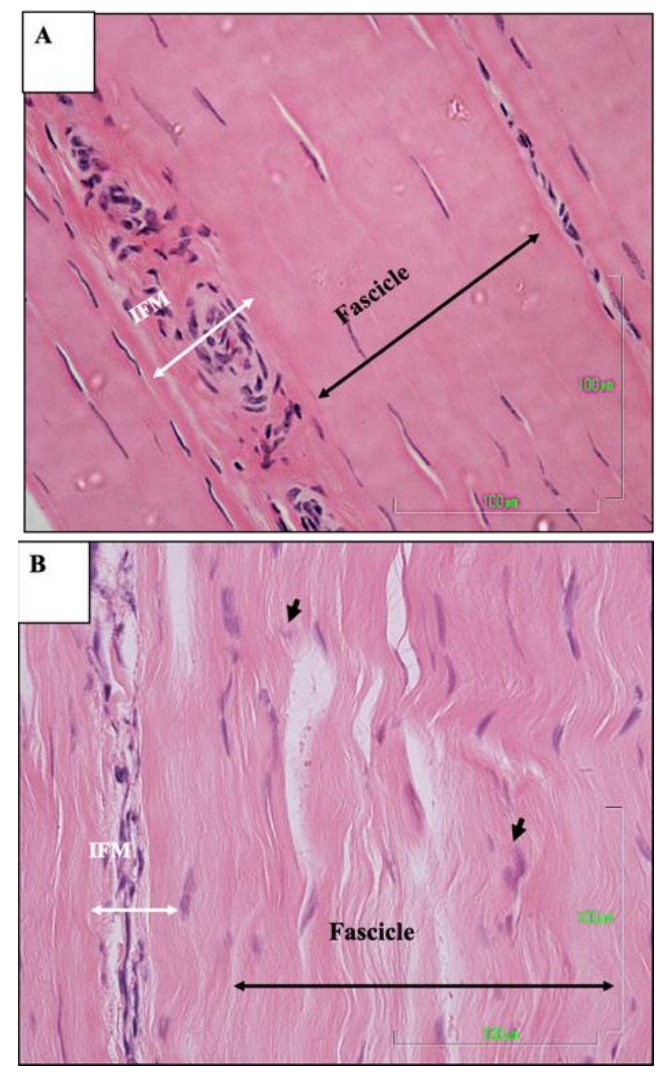

Figure 1: A; Longitudinal sections from the intact equine SDFT, show bundle of longitudinally oriented fibers named fascicles that separated by the IFM. The cells are regularly distributed between the collagen fibers of the fascicles and irregularly oriented within the IFM. B; Longitudinal section from the injured SDFT, which demonstrates disrupted collagen fibers of the fascicles with their different shaped intra-fascicular nuclei that arranged from short oval to a longer fusiform shaped with the apoptotic nucleuses (arrow heads). H\&E stained, (400 X).

In the injured SDFTs there were increased in the intensity of decorin immunostaining in both the fascicles and the IFMs. Although, all the injured samples showed upregulation of decorin immunostaining, but regional variation between IFMs and the fascicles were also observed. Nevertheless, the degree of decorin expression was slightly varied between different individual cases but they were not statistically significant. Interestingly, all the injured fascicles showed significant up-regulation of decorin immunostaining when compared to the normal fascicles. Statistically, fascicles from the injured tendons showed a significant increase in the degree of decorin expression, where the average decorin intensity scoring was $4.08( \pm 0.64 \mathrm{SD})$ when compared to normal fascicles (1.6 $\pm 0.37 \mathrm{SD})$. It was observed that during injured decorin was extensively expressed, which was about three folds when compared to 
the fascicles of the intact SDFTs (Figure 4). Further, regional variation of decorin intensity between the fascicles and IFMs were found within the injured SDFTs, the degree of decorin expression was also increased within the IFMs but it was not significant. The average immunostaining scoring of decorin of the IFM from the injured tendon was 3.5 ( $\pm 0.16 \mathrm{SD}$ ), which was greater than the normal IFMs but it was not significant statistically, using un-paired $\mathrm{T}$ test with a $\mathrm{P}$ value $<0.5$ (Figure 5).

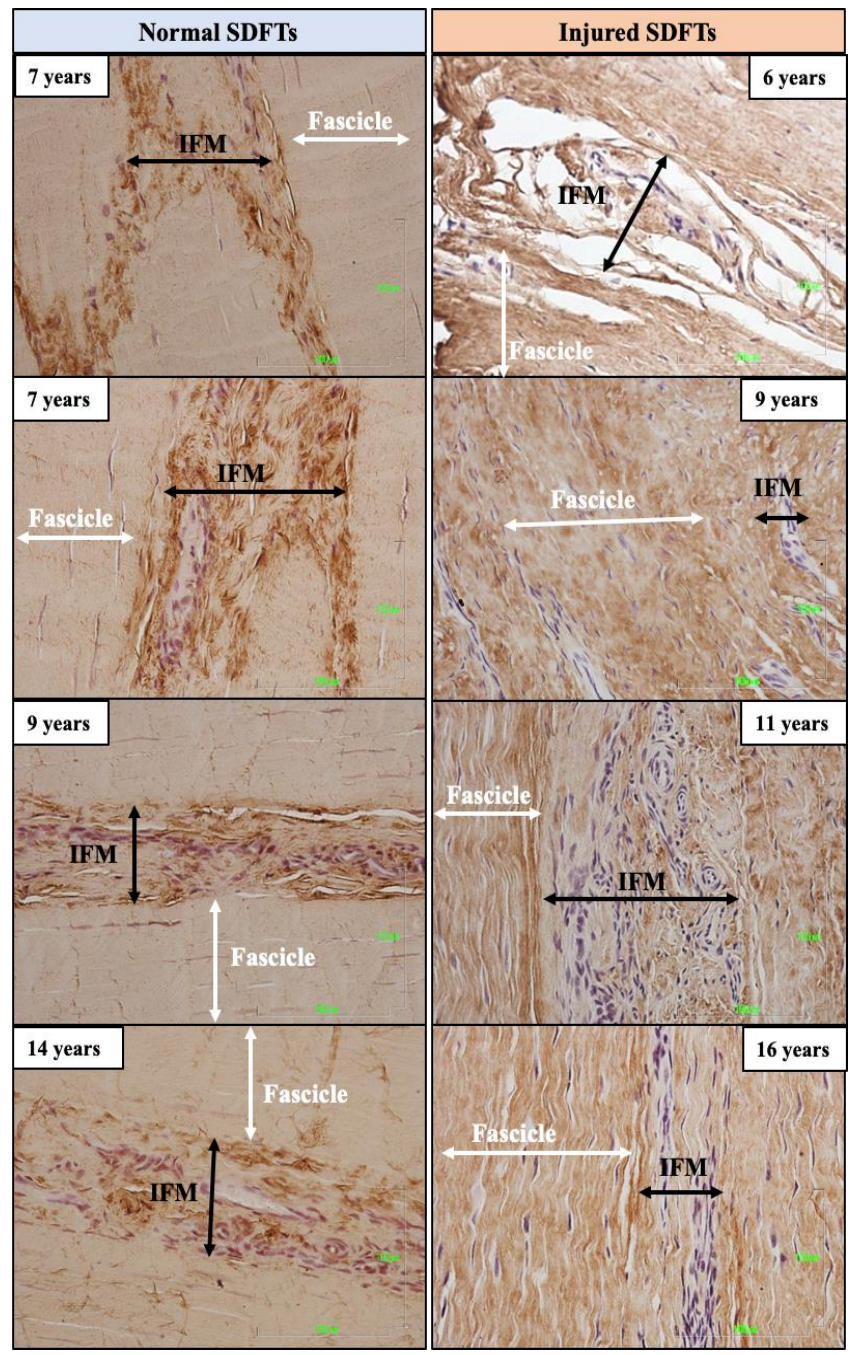

Figure 2: Immunostaining of the longitudinal sections of intact and the injured SDFTs from different ages with mouse monoclonal anti-decorin-IgG. They showed different intensity reactions to the monoclonal anti-decorin-IgG regionally in the normal and injured SDFTs, the injured SDFTs were shown intensive reaction for decorin, (400 X).

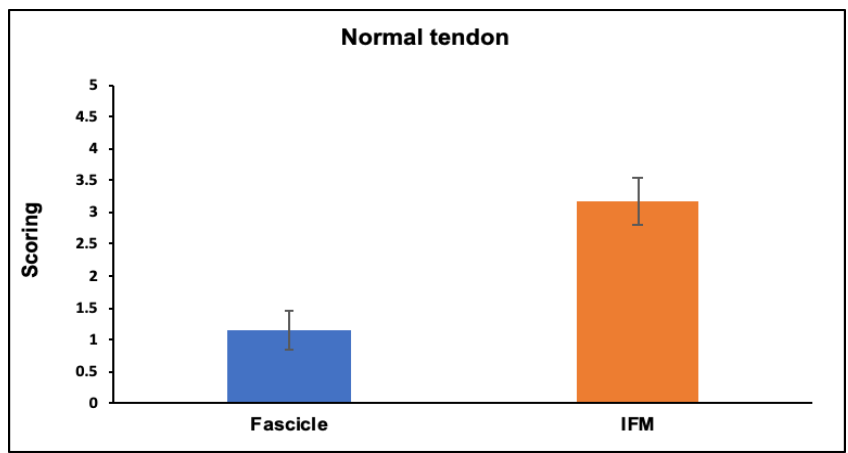

Figure 3: Show the average immunostaining intensity of decorin between the IFMs and fascicles. There was a significant difference in the intensity of decorin distribution between the IFMs and the fascicles, using un-paired T test, $(\mathrm{P}$ value $<0.05),($ Error bars $=\mathrm{SD})$, Graphpad prism version 5 .

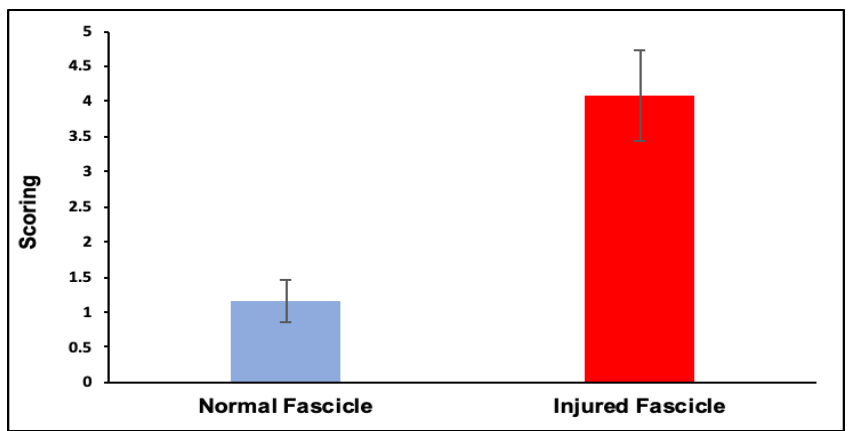

Figure 4: Show the average density of decorin between injured the normal fascicles. There was a significant difference in the density of decorin expression within the injured fascicles than the normal fascicles, using un-paired $\mathrm{T}$ test, $(\mathrm{P}$ value $<0.05)$, $($ Error bars $=\mathrm{SD})$, Graphpad prism version 5.

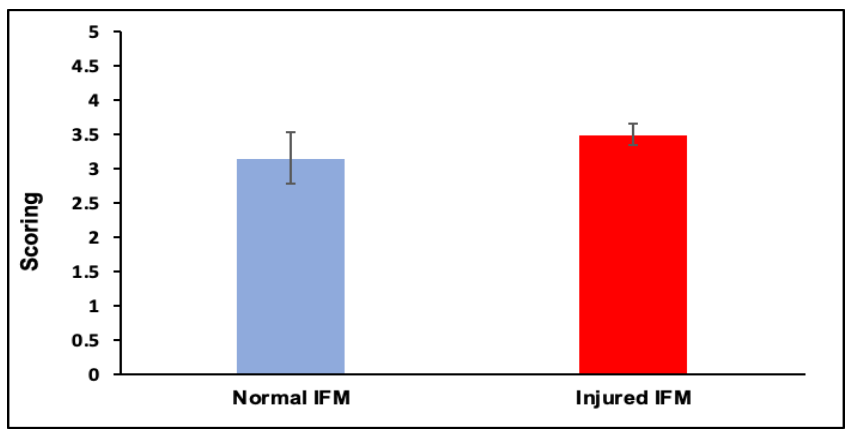

Figure 5: Show the average density of decorin between the injured and normal IFMs. There was not a significant difference in the density of decorin expression within the injured IFMs compared to the normal IFMs, using un-paired $\mathrm{T}$ test, $(\mathrm{P}$ value $<0.05)$, (Error bars $=\mathrm{SD})$, Graphpad prism version 5 . 


\section{Discussion}

In this study, specimens from intact and the injured SDFTs were examined for decorin immunostaining in order to understand regional variation in the distribution of this type of PG, because it is considered to be the most abundant SLRP of the tendon. It is acts as a key regulator in the process of fibrillogenesis, fibre alignment, fibrils inter-connection, stimulate cell proliferation and enhances immune responses within the tissue matrices $(12,14)$. In the current study, immunolocalization of decorin with mouse monoclonal $\mathrm{IgG}$ were showed regional variation in decorin level in the intact and injured tendons. Initially, normal SDFT samples were assigned for decorin immunostaining and they showed irregularly distributed decorin molecules within the normal tendon matrices. The IFM showed a greater amount of decorin localization rather than the fascicles, which may be attributed to the structure composition and the presence of the larger number of irregularly arranged cells. As well as, it has been reported that decorin is produced by different cells such as fibroblasts, chondrocytes, endothelial cells and smooth muscle cells, as they were more abundant in the IFMs rather than the fascicles in the intact tendons $(22,23)$. In addition to the collagen fibers binding, it is also interacts with fibronectin, thrombospondin, the complement component C1q, epidermal growth factor receptor (EGFR) and transforming growth factor-beta (TGF-beta) that directly influence the process of fibrillogenesis (24). Similarly, in an immunohistochemical study on the normal equine SDFT, they were recorded that decorin is one of the most prominent PGs, were localized within IFMs and the fascicles, but they did not record a significant regional variation between IFMs and the fascicles (8). Although, in an immunohistological study on decorin determination on the canine anterior cruciate ligaments (ACL) and the lateral digital extensor tendon (LDET), they were showed that decorin is distributed equally between IFMs and the fascicles in contrast to the current study (25). This variation in the intensity of decorin accumulation within the IFMs might be attributed to the structure conformation of the IFM and the presence of large number of cells. These cells are responsible for the production of various extracellular matrices such as decorin, as they are shown in different studies that PGs and GAG are present within the cytoplasm of the cells (intra-cellular), on cell membrane and within the connective tissues matrices $(26,27)$. Interestingly, in this study, it was recorded a significant amount of decorin was distributed around the cellular elements, collagen fibrils and the walls of blood vessels of the IFMs, as well as it was up-regulated in all samples were collected from injured tendons.

Different studies, showed that during tendinopathy the fascicles and IFMs lost their normal histological architecture, as well as other extracellular molecules are not remained constant particularly PGs and GAGs $(28,29)$. In the current clinical study, in addition to the histological alteration it was found that decorin was up-regulated in fascicles and the IFMs, although, the degree of up-regulation was varied when compared to the SDFT samples were collected the normal tendons. In the wounded tendons there were significant up-regulation of decorin within the fascicles rather than the IFM. Similarly, in an experimentally induced tendinopathy in equine flexor and extensor tendons, it was documented that decorin alone and GAGs such as chondroitin sulphate and dermatan sulphate were increased in the tendinopathic samples $(18,30)$. Meanwhile, the level of dermatan sulphate was up-regulated in both acute and chronic injury of SDFT but chondroitin sulphate was downregulated during acute injury of the SDFT. Thus, dermatan sulphate is considered to be important in binding decorin to the collagen fibrils to enhance the process of fibrogenesis and tissue regeneration $(31,32)$. In literatures, it has been shown that decorin up regulation exhibits an antiangiogenic activity, which provides a potential basis for the development of decorin-based therapies in these pathological situations particularly in a foreign body reactions and scarring tissue pathology (33). These upregulation of decorin with their GAGs (chondroitin and dermatan sulphate) chains indicating the potency of them in the process of tendon healing, because dermatan sulphate fasten to the surface of collagen fibrils molecules, where it act to regulates collagen fibre congregation and enhances both the lateral and longitudinal axial extension of the collagen fibres during acute and chronic tendinopathy $(34,35)$. On the other hand, it is well known that chondroitin sulphate furnishess tissue matrices strength against damaging via binding to the water molecules, which subsequently form hydrated matrices that provide cellular migration, tissue fibrils lubrication and enhancing the process of fibrillogenesis $(22,36,37)$. Nevertheless, during injury four kinds of cytokines (IL1- $\alpha$, IL1- $\beta$, TNF $\alpha$ and IFN $\gamma$ ) are also elevated variously in injured equine SDFT, they improve healing process $(38,39)$. Nevertheless, different components were used for the treatment of tendinopathy, such as corticosteroid, non-steroidal antiinflammatory drugs (NSAID), polysulfated glycosaminoglycan, hyaluronic acid (HA), platelet-rich plasma, TNF- $\alpha$, stem cell therapy and the surgical intervention (40). Interestingly, an extract in a form of Aloe vera gel were used in experimentally incised SDFT in donkey, it was found that the Aloe vera gel act as a protective cover over the wound and prevent wound contamination, which subsequently accelerated tendon healing $(41,42)$. In a current comparative experimental study in rabbits' model, Kessler sutures and polypropylene mesh were used in order to enhance healing of a severed calcaneal tendon. On the bases of clinico-pathological evaluation it was found that both techniques enhanced healing of the severed tendons but the mesh showed superiority over the suture group (43). In another recent study on experimentally severed Achilles tendon in dogs' model, were treated with tendorrhaphy, 
tendorrhaphy with platelet-rich plasma, tendorrhaphy with a vein graft, and tendorrhaphy and augmented with vein and platelet-rich plasma. It was found that using platelet-rich plasma with or without vein graft were enhanced tendon healing and improve its mechanical function (44). Therefore, it is required to consider that PGs and the GAGs apparently influence the tendon matrix components during tendinopathy through their effects in making the tendon microfibrils to counteract against further tissue destruction, as well as enhance the process fibrils re-organization and remodel the decayed tissue. This study showed the potential effect of decorin in the intact and injured SDFTs, although, the pathophysiological process of wound healing is not completely understood up to date $(45,46)$. This great expression of decorin during tendinopathy will indicate that the decorin is always associated with the collagen turnover in the intact and injured SDFTs, application of such PG in the clinical fields for the treatment of tendinopathy it must be considered and supported by further investigation (47). Meanwhile different clinical studies were performed on tendonitis including avian species (48).

\section{Conclusion}

It was demonstrated that the intensity of decorin immunolocalization were varied regionally, where the IFMs showed a significant amount of decorin when compared to the fascicles. In contrast to the normal SDFT, decorin immunostaining was presented a significant up-regulation in the injured fascicles when compared to the intact SDFT. This regional alteration to decorin indicates a greater fibril binding capacity, which make the tissue to sustain microdamages.

\section{Conflict of interest}

The authors declare that this paper it does not have conflict of interest.

\section{Acknowledgment}

The authors would like to thank the department of surgery and the veterinary teaching hospital of the college of veterinary medicine, university of Sulaimani for their support and accomplishing this clinical study.

\section{References}

1. Kastelic J, Galeski A, Baer E. The multicomposite structure of tendon. Connective Tissue Res. 1978;6:11-23. DOI: $10.3109 / 03008207809152283$

2. Kannus P. Structure of the tendon connective tissue. Scand J Med Sci Sports. 2000;10:312-20. DOI: 10.1034/j.1600-0838.2000.010006312

3. Benjamin M, Kaiser E, Milz S. Structure-function relationships in tendons:a review. J Anat. 2008;212:211-28. DOI: 10.1111/j.1469$\underline{7580.2008 .00864}$
4. Patterson-Kane JC, Rich T. Achilles tendon injuries in elite athletes:lessons in pathophysiology from their equine counterparts. ILAR Journal. 2014;55:86-99. DOI: 10.1093/ilar/ilu004

5. Thorpe CT, Birch HL, Clegg PD, Screen HR. The role of the noncollagenous matrix in tendon function. Int J Exp Pathol. 2013;94:24859. DOI: 10.1111/iep.12027.Epub

6. Halper J. Proteoglycans and diseases of soft tissues. Adv Exp Med Biol. 2014;802:49-58. DOI: 10.1007/978-94-007-7893-1_4

7. Sandell LJ. Molecular Biology of Proteoglycans and Link Proteins. In:Wight TN, Mecham RP, editors. Biology of Proteoglycans:Academic Press;1987. 27-57 p. DOI: 10.1016/C2013-011703-5

8. Thorpe CT, Karunaseelan KJ, Ng Chieng Hin J, Riley GP, Birch HL, Clegg PD, Hazel RC. Distribution of proteins within different compartments of tendon varies according to tendon type. J Anat. 2016:229:450-8 DOI: 10.1111/joa.12485

9. Evanko SP, Vogel KG. Ultrastructure and proteoglycan composition in the developing fibrocartilaginous region of bovine tendon. Matrix Bio. 1990;10:420-36. DOI: 10.1016/s0934-8832(11)80150-2

10. Rees SG, Dent CM, Caterson B. Metabolism of proteoglycans in tendon. Scand J Med Sci Sports. 2009;19:470-8. DOI: 10.1111/j.1600$\underline{0838.2009 .00938}$

11. Samiric T, Ilic MZ, Handley CJ. Characterisation of proteoglycans and their catabolic products in tendon and explant cultures of tendon. Matrix Bio. 2004;23:127-40. DOI: 10.1016/j.matbio.2004.03.004

12. Iozzo RV. The biology of the small leucine-rich proteoglycans. Functional network of interactive proteins. J Biol Chem. 1999;274:18843-6. DOI: $10.1074 / j b c .274 .27 .18843$

13. Samiric T, Ilic MZ, Handley CJ. Large aggregating and small leucinerich proteoglycans are degraded by different pathways and at different rates in tendon. Eur J Biochem. 2004;271:3612-20. DOI: 10.1111/j.0014-2956.2004.04307.x

14. Vesentini S, Redaelli A, Montevecchi FM. Estimation of the binding force of the collagen molecule-decorin core protein complex in collagen fibril. J Biomech. 2005;38:433-43. DOI: 10.1016/j.matbio.2004.03.004

15. Clegg PD, Strassburg S, Smith RK. Cell phenotypic variation in normal and damaged tendons. Int J Exp Pathol. 2007;88:227-35. DOI: 10.1111/j.1365-2613.2007.00549

16. Sodersten F, Hultenby K, Heinegard D, Johnston C, Ekman S. Immunolocalization of collagens (I and III) and cartilage oligomeric matrix protein in the normal and injured equine superficial digital flexor tendon. Connect Tissue Res. 2013;54:62-69. DOI: 10.3109/03008207.2012.734879

17. Parkinson J, Samiric T, Ilic MZ, Cook J, Handley CJ. Involvement of proteoglycans in tendinopathy. J Musculoskelet Neuronal Interact. 2011;11:86-93. DOI: 10.1111/j.1600-0838.2009.00938

18. Cadby JA, David F, van de Lest C, Bosch G, van Weeren PR, Snedeker JG, Van Schie HT. Further characterisation of an experimental model of tendinopathy in the horse. Equine Vet J. 2013;45:642-8. DOI: $\underline{10.1111 / \text { evj.12035 }}$

19. Jacobsen E, Dart AJ, Mondori T, Horadogoda N, Jeffcott LB, Little CB, Margaret MS. Focal Experimental Injury Leads to Widespread Gene Expression and Histologic Changes in Equine Flexor Tendons. PloS one. 2015;10:1-25. DOI: 10.1371/journal.pone. 0122220

20. Suvarna KS, Layton C, Bancroft JD. Bancroft's Theory and Practice of Histological Techniques: $8^{\text {th }}$ ed. Elsevier Health Sciences. 2019. 557 p.

21. Ali O. A descriptive study of the ultrastucture of the equine superficial digital flexor tendon [Ph.D. dissertation]. University of Liverpool. 2016. $310 \mathrm{p}$.

22. Rees SG, Flannery CR, Little CB, Hughes CE, Caterson B, Dent CM. Catabolism of aggrecan, decorin and biglycan in tendon. Biochem $\mathrm{J}$. 2000;350:181- 8. DOI: 10.1042/bj3500181

23. Kim B, Yoon JH, Zhang J, Eric Mueller PO, Halper J. Glycan profiling of a defect in decorin glycosylation in equine systemic proteoglycan accumulation, a potential model of progeroid form of Ehlers-Danlos syndrome. Arch Biochem Biophys. 2010;501:221-31. DOI: $\underline{10.1016 / \mathrm{j} . \mathrm{abb} .2010 .06 .017}$ 
24. Schönherr E, Broszat M, Brandan E, Bruckner P, Kresse H. Decorin Core Protein Fragment Leu155-Val260 Interacts with TGF- $\beta$ but Does Not Compete for Decorin Binding to Type I Collagen. Arch Biochem Biophys. 1998;355:241-8. DOI: 10.1006/abbi.1998.0720

25. Kharaz YA, Canty-Laird EG, Tew SR, Comerford EJ. Variations in internal structure, composition and protein distribution between intraand extra-articular knee ligaments and tendons. J Anat. 2018;232:94355. DOI: $10.1111 /$ joa. 12802

26. Silbert JE. Structure and metabolism of proteoglycans and glycosaminoglycans. J Invest Dermatol. 1982;79:31-37. DOI: 10.1111/1523-1747.ep12545094

27. Caterson B, Mahmoodian F, Sorrell JM, Hardingham TE, Bayliss MT, Carney SL, Ratcliffe A, Muir H. Modulation of native chondroitin sulphate structure in tissue development and in disease. J Cell Sci. 1990;97:411-7. DOI: $10.1242 /$ jcs. 97.3 .411

28. Van Schie HT, Bakker EM, Jonker AM, van Weeren PR. Computerized ultrasonographic tissue characterization of equine superficial digital flexor tendons by means of stability quantification of echo patterns in contiguous transverse ultrasonographic images. Am J Vet Res. 2003;64:366-75. DOI: $10.2460 /$ ajvr.2003.64.366

29. Meghoufel A, Cloutier G, Crevier-Denoix N, de Guise JA. Ultrasound B-scan image simulation, segmentation, and analysis of the equine tendon. Med Phys. 2010;37:1038-46. DOI: 10.1118/1.3292633

30. Smith MM, Sakurai G, Smith SM, Young AA, Melrose J, Stewart CM, Appleyard RC, Peterson JL, Gillies RM, Dart AJ, Sonnabend DH, Little $\mathrm{CB}$. Modulation of aggrecan and ADAMTS expression in ovine tendinopathy induced by altered strain. Arthritis Rheum. 2008;58:105566. DOI: $10.1002 /$ art.23388

31. Mori Y. Inflammation and proteoglycans. In:M. Muramatsu and K. Nagai, editors. New Experimental Biochemistry. 1st Ed. Tokyo Kagaku Dohjin, Tokyo.1991.442-448 p.

32. Kobayashi A, Sugisaka M, Takehana K, Yamaguchi M, Eerdunchaolu, Iwasa EK, Abe M. Morphological and histochemical analysis of a case of superficial digital flexor tendon injury in the horse. J Comp Pathol. 1999;120:403-14. DOI: $10.1053 /$ jcpa.1998.0288

33. Järveläinen $\mathrm{H}$, Sainio $\mathrm{A}$, Wight TN. Pivotal role for decorin in angiogenesis. Matrix Bio. 2015;43:15-26. DOI: 10.1016/j.matbio.2015.01.023

34. Birk DE, Nurminskaya MV, Zycband EI. Collagen fibrillogenesis in situ:fibril segments undergo post-depositional modifications resulting in linear and lateral growth during matrix development. Dev Dyn. 1995;202:229-43. DOI: 10.1002/aja.1002020303

35. Silver FH, Freeman JW, Seehra GP. Collagen self-assembly and the development of tendon mechanical properties. J Biomech. 2003;36:1529-53. DOI: 10.1016/s0021-9290(03)00135-0

36. Vogel KG, Heinegard D. Characterization of proteoglycans from adult bovine tendon. J Biol Chem. 1985;260:9298-306. DOI: 10.1016/S0021-9258(17)39366-3

37. Martinez P, Denys A, Delos M, Sikora AS, Carpentier M, Julien S, Pestel J, Allain F. Macrophage polarization alters the expression and sulfation pattern of glycosaminoglycans. Glycobiology. 2015;25:50213. DOI: $10.1093 /$ glycob/cwu 137

38. Hosaka Y, Kirisawa R, Yamamoto E, Ueda H, Iwai H, Takehana K. Localization of cytokines in tendinocytes of the superficial digital flexor tendon in the horse. J Vet Med Sci. 2002;64:945-7. DOI: 10.1292/jvms.64.945

39. Dakin SG, Werling D, Hibbert A, Abayasekara DR, Young NJ, Smith RK, Dudhia J. Macrophage sub-populations and the lipoxin A4 receptor implicate active inflammation during equine tendon repair. PLoS ONE. 2012;7:32333-45. DOI: 10.1371/journal.pone.0032333

40. Ho JO, Sawadkar P, Mudera V. A review on the use of cell therapy in the treatment of tendon disease and injuries. J Tissue Eng. 2014;5:1-18. DOI: $10.1177 / 2041731414549678$

41. R. Al-Kennany E, H. Allawi A, T. Abd-Almaseeh Z. Effect of aloe vera gel on experimantal tendon healing in donkeys. Iraqi $\mathrm{J}$ Vet Sci. 2006;20:173-80. DOI: 10.33899/ijvs.2006.62507

42. Docheva D, Müller SA, Majewski M, Evans CH. Biologics for tendon repair. Adv Drug Deliv Rev. 2015;84:222-39. DOI: 10.1016/j.addr.2014.11.015
43. Humadi S. A comparative study between kessler suture versus polypropylene mesh implantation to repair tenotomized common calcaneal tendon in rabbits. Iraqi J Vet Sci. 2019;33:289-96. DOI: 10.33899/ijvs.2019.162907

44. Allawi AH, Alkattan lm, aliraqi om. Clinical and ultrasonographic study of using autogenous venous graft and platelet-rich plasma for repairing Achilles tendon rupture in dogs. Iraqi J Vet Sci. 2019;33:453-60. DOI: 10.33899/ijvs.2019.163199

45. Watts AE, Yeager AE, Kopyov OV, Nixon AJ. Fetal derived embryoniclike stem cells improve healing in a large animal flexor tendonitis model. Stem Cell Res Ther. 2011;2:27-57. DOI: $10.1186 / \mathrm{scrt} 45$

46. Veronesi F, Della BE, Torricelli P, Pagani S, Fini M. Effect of adiposederived mesenchymal stromal cells on tendon healing in aging and estrogen deficiency:an in vitro co-culture model. Cytotherapy. 2015;17:1536-44. DOI: $10.1016 /$ j.jcyt.2015.07.007

47. Robinson KA, Sun M, Barnum CE, Weiss SN, Huegel J, Shetye SS, Lin L, Saez D, Adams SM, Iozzo RV, Soslowsky LJ, Birk DE. Decorin and biglycan are necessary for maintaining collagen fibril structure, fiber realignment, and mechanical properties of mature tendons. Matrix Bio. 2017;64:81-93. DOI: 10.1016/j.matbio.2017.08.004

48. Isolation and detection of reovirus from arthritis in chickens. Iraqi J Vet Sci. 2020;34:59-63. DOI: 10.33899/ijvs.2019.125580.1093
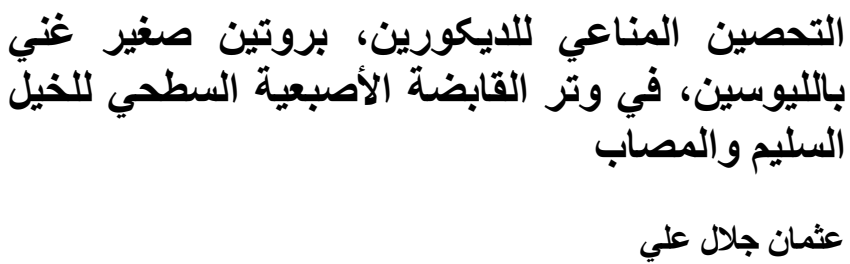

فرع الجراحة والتناسل، كلية الطب البيطري، جامعة السليمانية، السليمانية، العراق

الخلاصة

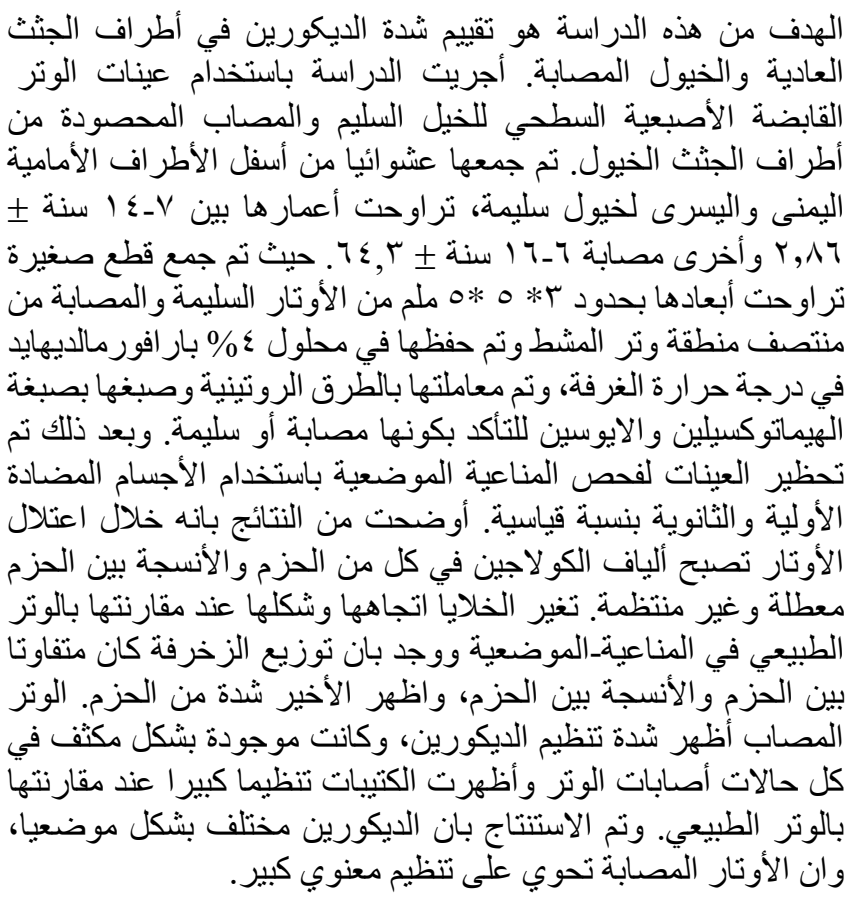

